\title{
e-Phaïstos
}

e-Phaïstos

Revue d'histoire des techniques / Journal of the history

of technology

IX-2 | 2021

Quel objet pour quel musée?

\section{Archéologie contemporaine de l'industrie}

Une étude de cas : La friche des Vaux de Vire (Calvados, France)

Contemporary Industrial Archeology. A Case Study: The Vaux de Vire's

Industrial Wasteland

Morgane Moëllo, Cyril Lacheze et Jérôme Féru

\section{OpenEdition}

\section{Journals}

Édition électronique

URL : https://journals.openedition.org/ephaistos/9225

DOI : 10.4000/ephaistos.9225

ISSN : 2552-0741

Éditeur

IHMC - Institut d'histoire moderne et contemporaine (UMR 8066)

Référence électronique

Morgane Moëllo, Cyril Lacheze et Jérôme Féru, « Archéologie contemporaine de l'industrie », ePhaïstos [En ligne], IX-2 | 2021, mis en ligne le 26 octobre 2021, consulté le 28 octobre 2021. URL : http://journals.openedition.org/ephaistos/9225; DOI : https://doi.org/10.4000/ephaistos.9225

Ce document a été généré automatiquement le 28 octobre 2021.

Tous droits réservés 


\title{
Archéologie contemporaine de l'industrie
}

\author{
Une étude de cas : La friche des Vaux de Vire (Calvados, France) \\ Contemporary Industrial Archeology. A Case Study: The Vaux de Vire's \\ Industrial Wasteland
}

Morgane Moëllo, Cyril Lacheze et Jérôme Féru

1 La friche de l'ancienne usine Société Générale d'Équipement (SGE) / Labinal se situe dans le département du Calvados, en aval de la ville de Vire, au cœur de la vallée industrielle des Vaux de Vire. Destinée à la production de pièces automobiles et aéronautiques, son activité s'est étendue de 1936 à 1985, avant un abandon du site. Au début des années 2010 , les autorités locales ont souhaité réaliser une étude historique et patrimoniale du site, afin d'envisager une reconversion documentée par l'histoire des lieux. Les trois co-auteurs ont effectué celle-ci sur deux semaines, en avril 2012 ; le rapport étant disponible en ligne ${ }^{1}$.

2 Au-delà de la volonté de documentation initiale, cette étude s'est voulue la plus exhaustive possible, abordant plusieurs problématiques : choix et solutions techniques déployées pour la construction des bâtiments, impacts environnementaux passés ou actuels, signification patrimoniale, ou encore pistes sociétales et sociologiques concernant l'occupation du lieu aux différentes époques de sa "vie », y compris en tant que friche et non plus en tant qu'usine fonctionnelle.

\section{Sources et méthodologie}

Nous avons en conséquence fait le choix d'effectuer cette étude dans une démarche de type « anthropologique » dans le sens anglo-saxon du terme, autrement dit en prenant en compte, autant que possible, tous les types de sources et méthodes d'analyse à disposition. Celles-ci incluent des sources textuelles et des enquêtes orales, habituelles de l'histoire récente, mais également les aspects matériels, à commencer par les bâtiments eux-mêmes. Le fait de considérer ceux-ci comme une source à part entière 
implique, au niveau disciplinaire, un recours à l'archéologie, en tant qu'étude du passé à partir des sources matérielles. L'historiographie correspondant est cependant pauvre en France, quoique existante dans le monde anglo-saxon, et la démarche de la présente étude parait particulièrement novatrice. Si le concept d'archéologie des périodes récentes a été présenté depuis au moins un siècle (Deonna 1922 : 45-46), l'archéologie des périodes moderne et contemporaine commence seulement (et à plus forte raison au moment de réalisation de l'étude) à se développer de façon autonome en France et à être reconnue comme telle (Journot et Bellan 2011; Huard, Roumégoux et ChaouiDerieux 2014 ; Bonnot 2018).

4 Même ainsi, les archéologues français ne s'intéressent pas pour l'instant à des bâtiments en place aussi récents que ceux de la SGE. Les trois dernières décennies ont certes vu l'émergence de "l'archéologie industrielle», particulièrement sous l'impulsion d'historiens des techniques (Daumas 1980). On peut se référer en particulier aux publications du CILAC: la revue L'Archéologie industrielle en France ayant par exemple publiée une première synthèse de la présente étude (Moëllo, Lacheze et Féru 2013). Toutefois, si l'intérêt pour les vestiges matériels est très présent dans cette démarche, la méthode employée n'est pas véritablement archéologique et reste basée sur des recherches en archives: le terrain fait, lui, massivement l'objet de photographies et de croquis, mais non de relevés précis et de réflexions s'appuyant en priorité sur ceux-ci.

5 Les relevés des bâtiments et des berges réalisés lors de la campagne, avec une méthodologie de relevé simplifiée s'approchant de celle proposée par Thomas Renk dès la fin de la décennie $1960^{2}$, fournissent de fait la documentation la plus dense : le planmasse des vestiges en élévation et des traces relevées au sol montre l'étendue de ceuxci (cf. fig.1). S'y ajoutent des sources textuelles de plusieurs provenances. Si les archives de l'entreprise à proprement parler n'ont pu être localisées (y compris dans les fonds propres de Safran, propriétaire actuel de l'ancienne SGE via plusieurs rachats et changements de noms, dont «Labinal»), les archives départementales du Calvados conservent tout de même des documents en rapport, tout comme l'Institut Géographique National pour les photographies aériennes.

Fig.1 : Plan-masse général du site

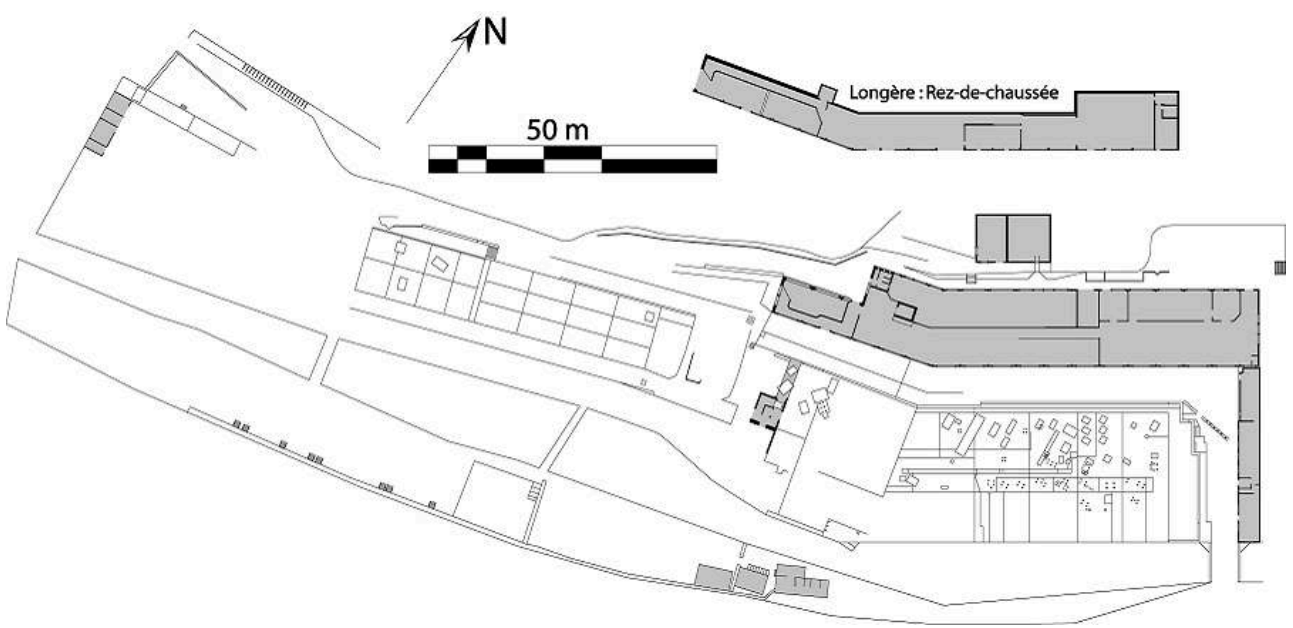

Les parties grisées sont celles demeurant en élévation. Le plan principal montre l'étage de la longère. Relevé Morgane Moëllo, Cyril Lacheze et Jérôme Féru ; DAO Cyril Lacheze 
Quelques autres sources ont été fournies par les Services Techniques de la ville de Vire et par les anciens employés. Surtout une importante documentation a été trouvée in situ, éparpillée sur le sol des bâtiments et déposée au musée de Vire après l'étude (cf. fig.2). Enfin, une rencontre des anciens employés de la SGE organisée par les municipalités a permis de réaliser une série d'entretiens fournissant une ébauche d'enquête sociologique.

Fig.2. Un des nombreux documents retrouvés sur place (calendrier de formations de 1973).

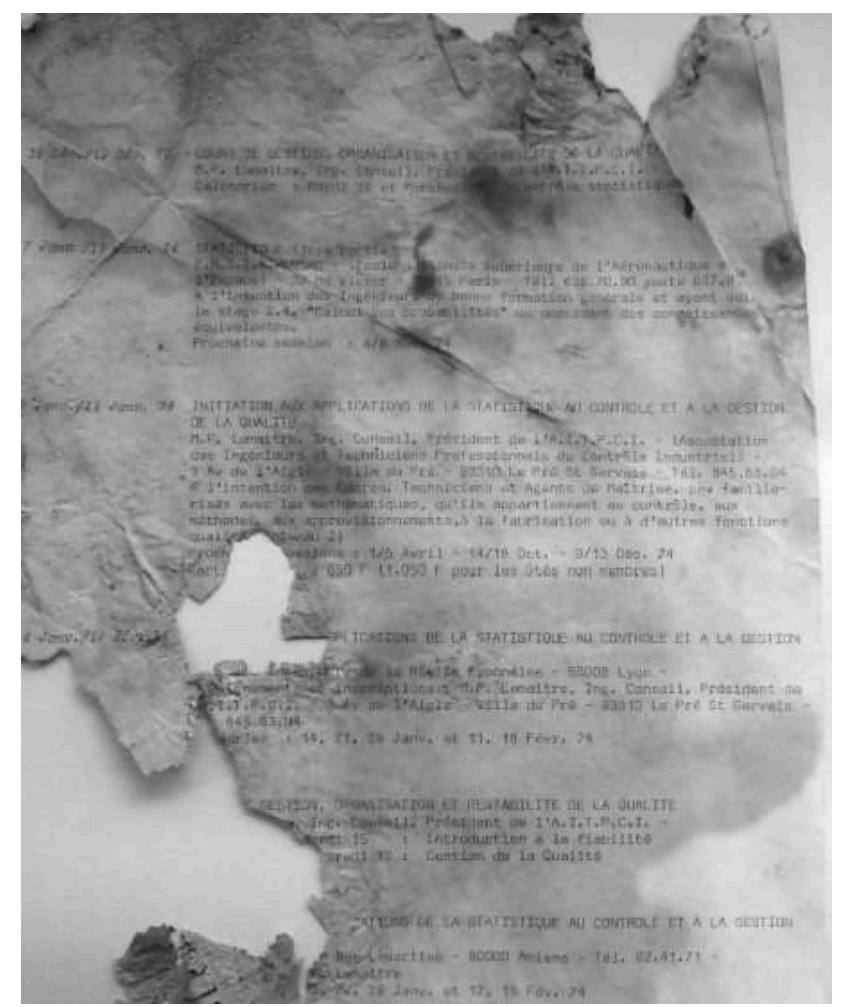

Morgane Moëllo

\section{Évolution générale du site}

\section{Historique de l'occupation}

7 La vallée des Vaux de Vire constitue un ensemble industriel depuis le XIX ${ }^{\mathrm{e}}$ siècle au moins, auquel participe pleinement le site de la friche industrielle. Le cadastre napoléonien figure en 1833 un ensemble architectural en pierres de taille, correspondant à une fabrique textile de draps ${ }^{3}$, lequel s'étendait de manière dispersée sur la totalité de la parcelle actuelle d'1,5 hectare en 1855 après un remembrement (cf. fig.3 $)^{4}$. Cette configuration spatiale perdura durant la seconde moitié du XIX ${ }^{\mathrm{e}}$ siècle, y compris lors du changement de spécialisation textile pour la bonneterie au début du $\mathrm{XX}^{\mathrm{e}}$ siècle, si ce n'est qu'un chemin communal traversait alors le terrain et que des habitations occupaient le nord du site. 
Fig. 3. Terrain d'implantation de l'usine en 1855 : fabrique de draps Juhel-Pondegrenne

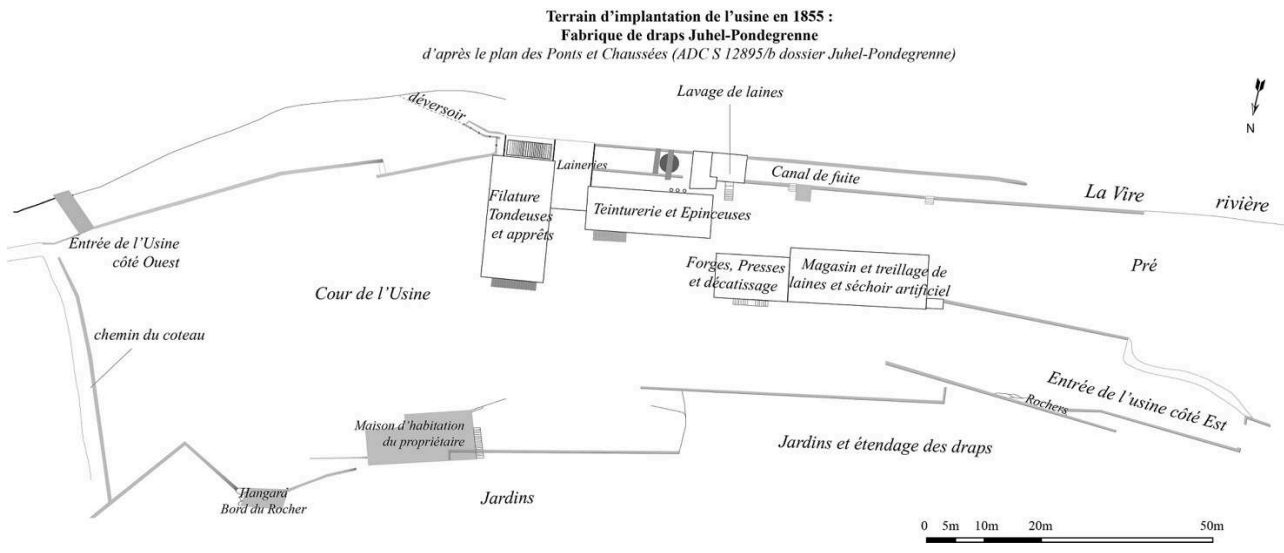

Archives départementales du Calvados, S 12896/a, 1855

Relevé et DAO Morgane Moëllo

8 Le seul vestige de cette époque identifié, probablement lié à la fabrique textile de la première moitié $\mathrm{du} \mathrm{XIX}^{\mathrm{e}}$ siècle, est un ensemble architectural en pierre de taille témoignant de la présence d'ouvrages hydrauliques. Sa situation correspond à l'aval d'un seuil, à un endroit où la Vire, pareillement à la longère (cf. infra) et à la falaise, forme un coude. Il se compose d'un plan incliné reposant sur deux pans de murs de la rive nord (cf. fig.4 et 5). Une cavité rectangulaire située dans la partie centrale du plan incliné, remplie d'une pièce de bois percée en son centre et de mortier, a pu correspondre à un support de fixation pour un étais de vannage.

Fig.4 : Barrage au niveau du plan incliné de l'ancienne fabrique textile

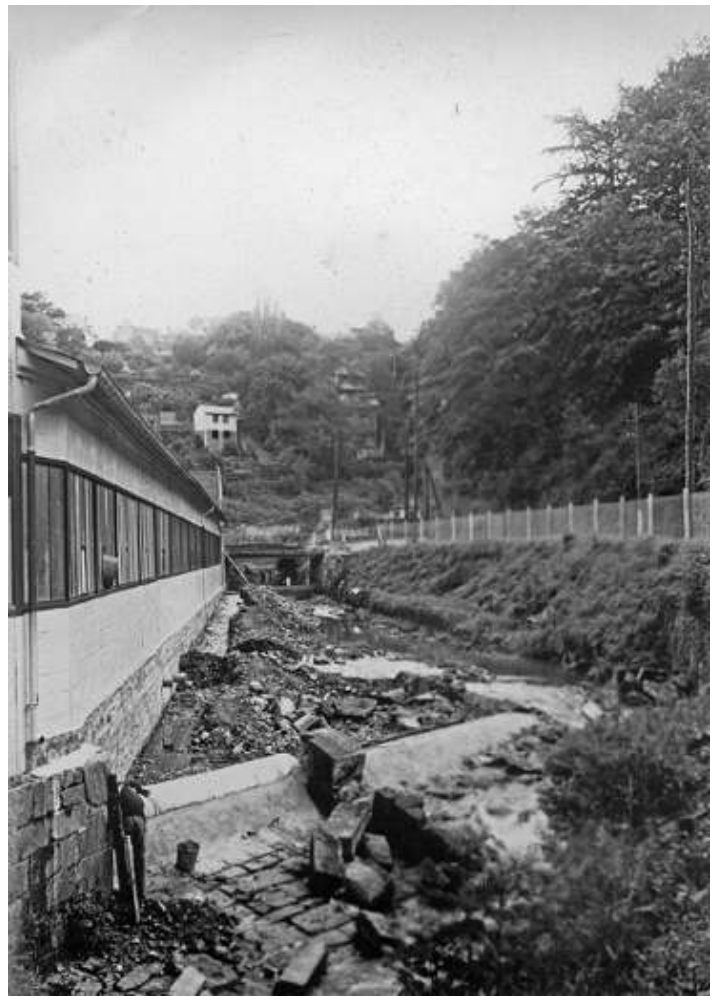

Vue prise durant les travaux de construction de la SGE, en 1937.

Courtoisie de Jean Jacques 
Le plan incliné aurait également pu servir au transport du bois, mais nous n'en avons pas mention dans les sources; c'est pourquoi l'hypothèse d'une optimisation de l'écoulement de l'eau sous une roue prend plus de poids. Un fragment de mur cernant le plan incliné au sud devait constituer un support de maintien du barrage contre le courant. Il s'agit là d'un aménagement hydraulique notable, susceptible d'être valorisé d'un point de vue patrimonial, considérant son intérêt pour la compréhension du site avant l'implantation de la SGE.

Fig.5 : Relevé du plan incliné.

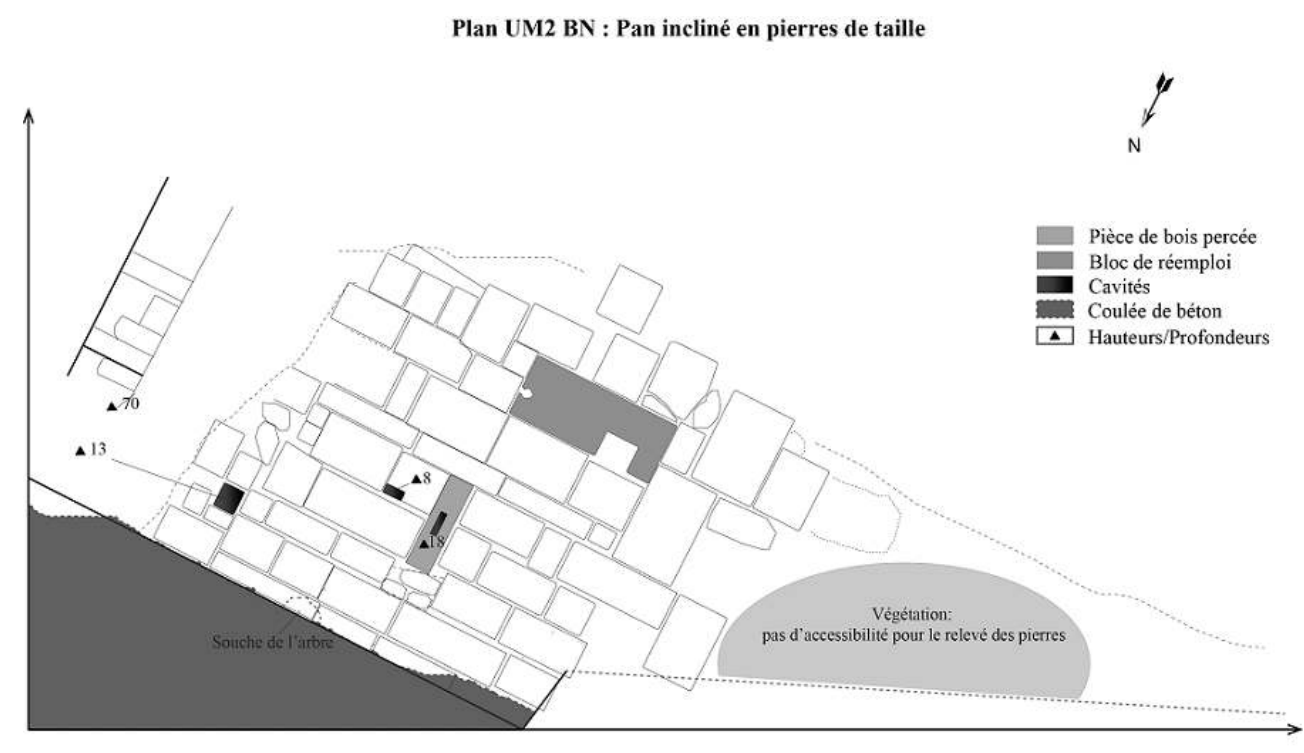

$0 \quad 50 \mathrm{~cm} 1 \mathrm{~m} \quad 2 \mathrm{~m} \quad 5 \mathrm{~m}$

Relevé Morgane Moëllo, Jérôme Féru et Cyril Lacheze ; DAO Morgane Moëllo

\section{Développement et aménagement des berges}

Les occupations du XIX ${ }^{\mathrm{e}}$ siècle ne semblent pas avoir modifié de manière drastique le lit mineur du cours d'eau. Toutefois, l'impact du contexte général d'ancrage du site au cœur de l'activité industrielle de la haute-vallée viroise n'est pas négligeable de ce point de vue, au sens où l'utilisation massive de l'énergie hydraulique et la lutte contre les inondations impliquèrent la canalisation progressive du tronçon urbain de la Vire, en cours mais encore inachevée lors de la construction de la SGE en 1937. Or, les élus expriment un intérêt pour la connaissance du linéaire fluvial antérieur à l'implantation de l'usine, en écho au contexte législatif européen actuel de restauration du bon état écologique des cours d'eau. Le relevé archéologique des deux berges a de fait mis en évidence des strates murales majoritairement schisto-gréseuses faisant état de l'usage des ressources géologiques locales et plusieurs remaniements.

11 La période d'exploitation du terrain par l'usine d'équipement marqua une rupture plus franche dans la manière d'occuper l'espace. Son installation impliqua en premier lieu le terrassement du site par le nivellement et le bétonnage afin de former la base des ateliers. Les bords du cours d'eau furent transformés en plate-forme solide apte à recevoir les unités de production : les sources iconographiques ainsi que la présence de couches de béton au-dessus des moellons des berges en témoignent. En amont, à 
l'entrée du site, la berge nord garde les traces du surhaussement de la berge et du pont par deux strates de moellons maçonnés, présentant deux sections de poutres arasées supports d'un ancien pont. En aval, un ancien canal de fuite, vestige des installations du $\mathrm{XIX}^{\mathrm{e}}$ siècle sur la rive nord, fut comblé par des blocs de schiste. Sur la berge sud, des traces des structures porteuses apparaissent également en négatif.

L'emprise spatiale de l'usine atteint son paroxysme aux alentours de 1945 et, comme le précisait le directeur Lucien Motion en $1963^{5}$, elle avait alors épuisé toutes ses possibilités d'extension. D'après les photographies aériennes de la décennie 1950, toutes les constructions préexistantes avaient été détruites ou réemployées: les coteaux des Rames au nord du site dynamités pour gagner de la place (et les blocs de schiste produits utilisés dans les bâtiments), des bâtiments construits dans le prolongement des murs de berges, et une passerelle couverte mise en place au-dessus de la Vire ${ }^{6}$. L'abandon du site en 1985 démontre que l'espace du site ne répondait plus à l'échelle de la production. Ainsi, l'impact environnemental de la SGE est indéniable, via la transformation volontaire et l'optimisation de l'espace disponible.

\section{Vestiges de l'usine}

\section{Terre-plein extérieur}

La majeure partie de la friche de la SGE est constituée d'un terre-plein bétonné, qui composait le sol des bâtiments industriels. À l'exception de celles décrites plus bas, la quasi-totalité des constructions a été totalement arasée; toutefois, il subsiste encore dans de nombreux cas la trace des cloisons ainsi que des emplacements de machines sur le sol. En utilisant ces données ainsi que le plan général du site dressé en $1961^{7}$, il est possible de reconstituer une partie de la chaîne de production de l'usine, montrant une organisation rationalisée et segmentée. Il apparaît que la construction des bâtiments en eux-mêmes ne correspond pas systématiquement à l'organisation des activités qui y prenaient place, là où, faute de documentation archéologique, l'on pourrait admettre par défaut qu'une unité architecturale correspond à une unité fonctionnelle.

Sur la rive sud de la Vire, les vestiges sont peu lisibles à cause de la végétation et d'une importance très relative : un transformateur et une réserve de fioul encore en place, quelques traces d'un bâtiment. Par contre, pour la berge nord correspondant au cœur de l'usine, une demi-douzaine de secteurs distincts a pu être repérée. Le plus lisible est l'atelier $\mathrm{A}$, correspondant à toute une zone jouxtant à la fois l'entrée, la rivière, la longère et le monte-charge, bien désherbée et parfaitement lisible (cf. fig.6). 
Fig.6 : Relevé des traces au sol

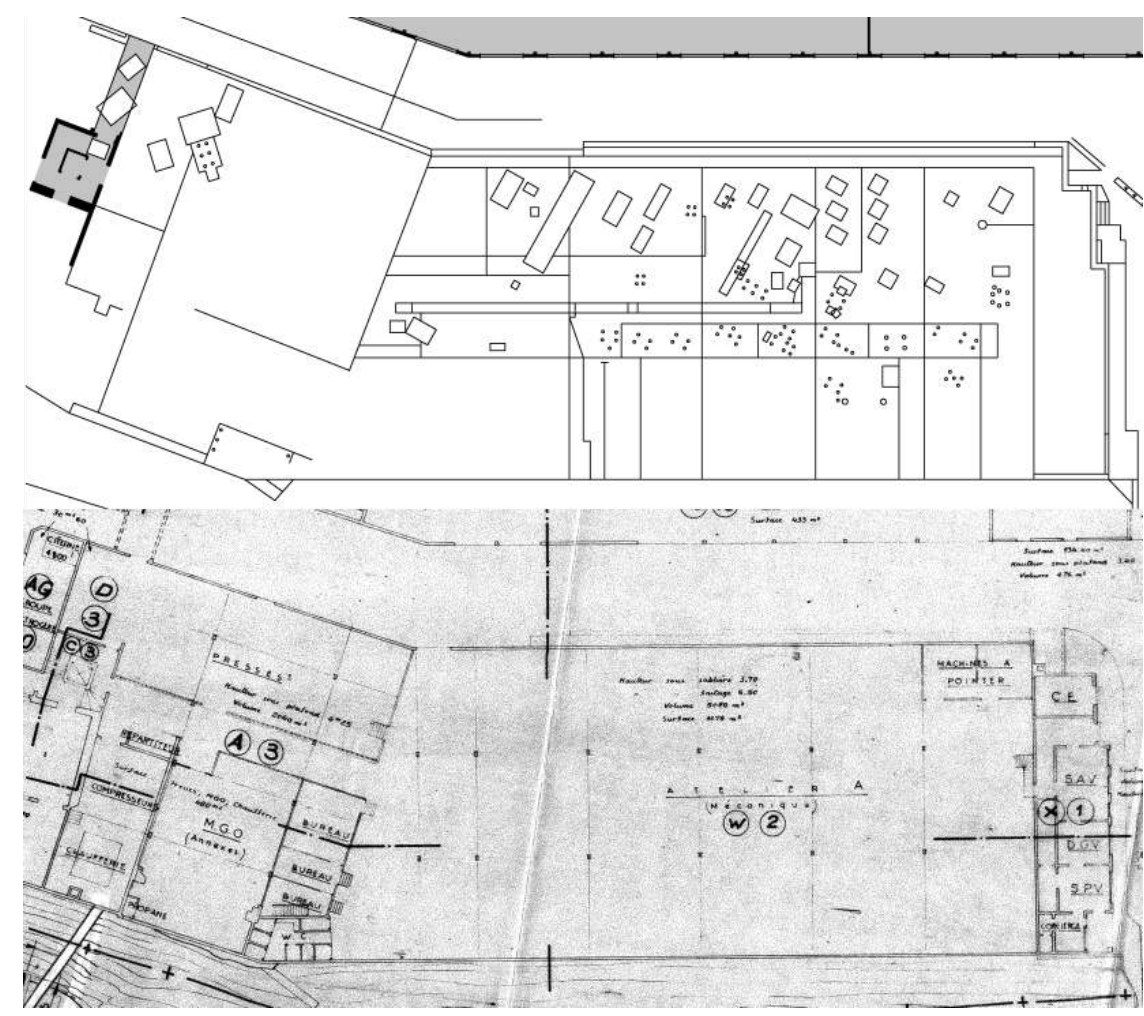

Relevé sur une partie du terre-plein et zone correspondante sur le plan-masse de 1961.

Relevé Jérôme Féru et Cyril Lacheze ; DAO Cyril Lacheze. Précision Mécanique Labinal, Usine des Vaux de Vire. Plan masse, 1961. Courtoisie des Services Techniques de la ville de Vire.

Le plan de 1961 en fait un «atelier mécanique » très largement ouvert, sans aucune séparation interne; pourtant, les traces au sol montrent une spécialisation de l'espace avec des regroupements de machines semblables, confirmés par l'enquête orale qui indique effectivement une telle logique. A contrario, à proximité immédiate du montecharge (grisé sur le relevé), une zone correspondant à des presses s'inscrit architecturalement dans un tout autre bâtiment, avec une orientation bien différente, mais est du point de vue organisationnel une annexe de l'atelier A. Cette dichotomie au moins partielle entre architecture et fonction se retrouve de manière générale dans une grande partie des secteurs extérieurs.

\section{Architecture et matériaux}

De l'ensemble de l'usine ne subsistent plus qu'un long corps de bâtiment au Nord-Est du terrain, que nous appelons la " longère " par commodité (environ 30 mètres de long sur 6 de large, et 7 de hauteur), quelques petites constructions à l'entrée du terrain, et un certain nombre d'annexes éparpillées. D'après l'étude de terrain et des photographies anciennes, toutes ces constructions ont été conçues spécifiquement pour l'usine et y sont donc adaptées dès l'origine. Les constructions à l'entrée de l'usine ne correspondent qu'à la loge du concierge, des pointeuses et un vestiaire, sans intérêt particulier que ce soit au plan organisationnel ou architectural.

La construction de la longère est beaucoup plus parlante, en particulier du point de vue de l'adaptation de bâtiments industriels préconçus voire en partie préfabriqués, à un 
terrain difficile. En effet, afin de maximiser l'espace, la longère est placée contre une falaise formant un coude, et impliquant une dénivellation à la fois du Nord au Sud et de l'Est à l'Ouest. Il n'a donc pas été possible pour l'architecte de considérer le terrain comme un espace vierge, mais il lui a fallu s'adapter à de nombreuses conditions spécifiques. La construction en elle-même était toutefois originellement normée, et manifestement prévue pour un terrain dégagé. La longère était en effet constituée de trois corps de bâtiments accolés en enfilade et a priori identiques, avec un espace intérieur totalement dégagé et aménageable à volonté.

Assez classiquement, la structure reposait sur six piliers porteurs en béton pour chaque façade, supportant une dalle également de béton servant de plancher à l'étage, ainsi que des fermes préfabriquées en acier, toutes identiques. L'intérieur de ces travées en façade n'était pas porteur et pouvait être comblé de quelque manière que ce soit, généralement en briques et comportant une grande fenêtre pour un éclairage maximisé. Les murs pignons de l'ensemble de la longère étaient par contre édifiés en moellons, sans que l'on puisse trouver une raison évidente à cela, à part peut-être le réemploi sur place de blocs précédemment arrachés par dynamitage à la falaise, et/ou provenant des constructions antérieures sur le site.

Fig.7 : Façade Sud de la longère

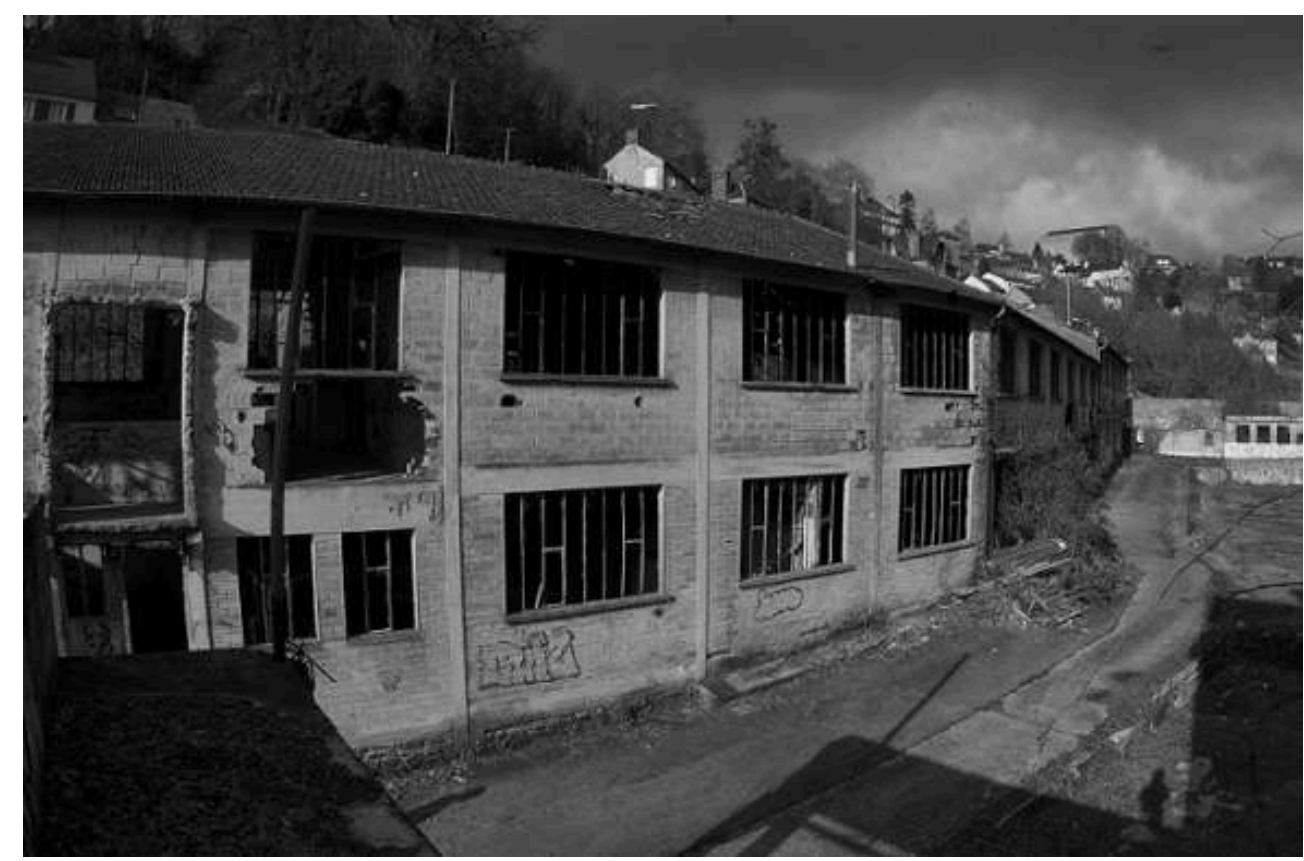

Photographie Alexis Seydoux

La plus évidente des adaptations effectuées à partir de ce modèle pour épouser la courbure et la pente du terrain tient au fait que, pour gagner de l'espace, le rez-dechaussée du bâtiment fut en partie creusé dans la falaise : le mur Nord était totalement aveugle, et se présentait sous la forme d'un simple parement de moellons apposé sur la roche naturelle. Ceci impliquait également une moitié nord de l'espace plus étroite que la moitié sud sur une grande partie de la construction, dans la longueur. Ainsi, l'étage se trouvait au rez-de-chaussée lorsqu'on y accédait depuis le Nord du terrain, contre la falaise, créant des possibilités de circulation supplémentaires. 

un radier de béton et de moellons, tandis que l'ensemble de celui de l'est était surélevé de 1,50 m par rapport aux deux autres pour ne pas s'enfoncer dans la roche. Ceci impliquait, à l'intérieur, la présence de deux rampes de plusieurs mètres de longueur (une à chaque étage) pour relier en douceur le bâtiment est et le bâtiment central, lesquelles présentaient une contrainte forte au niveau de l'agencement spatial, même si l'espace alentour était organisé en conséquence. Enfin, le bâtiment à l'ouest de la longère formait un angle d'une trentaine de degrés par rapport aux autres, afin d'épouser la courbe de la falaise, sans que cela n'entraîne de contrainte forte cette fois.

En-dehors de la longère, le principal élément encore en place est le monte-charge, faisant initialement partie d'un autre bâtiment. Un certain nombre de fragments de murs d'aspect plus ancien semblent s'y rattacher, mais la tour elle-même présente une conception similaire à celle de la longère : une structure porteuse, cette fois composée de poutres en acier, remplie par des briques, des fenêtres ou des portes selon les besoins (cf. fig.8). Là encore, le terrain est pentu et aménagé en conséquence, avec une entrée à un niveau différent selon le côté considéré. Enfin, outre le transformateur en béton de la rive Sud, la seule autre construction est une réserve à charbon flanquée d'une maisonnette à l'utilité inconnue, accrochée à flanc de falaise et édifiée presque entièrement en moellons; elle est toutefois sans doute contemporaine de l'usine puisque le terrain est mis à niveau par une dalle de béton. Il ne s'agit donc pas d'un réemploi comme la construction moins « industrielle » pourrait le faire penser.

Fig. 8 : Façades du monte-charge
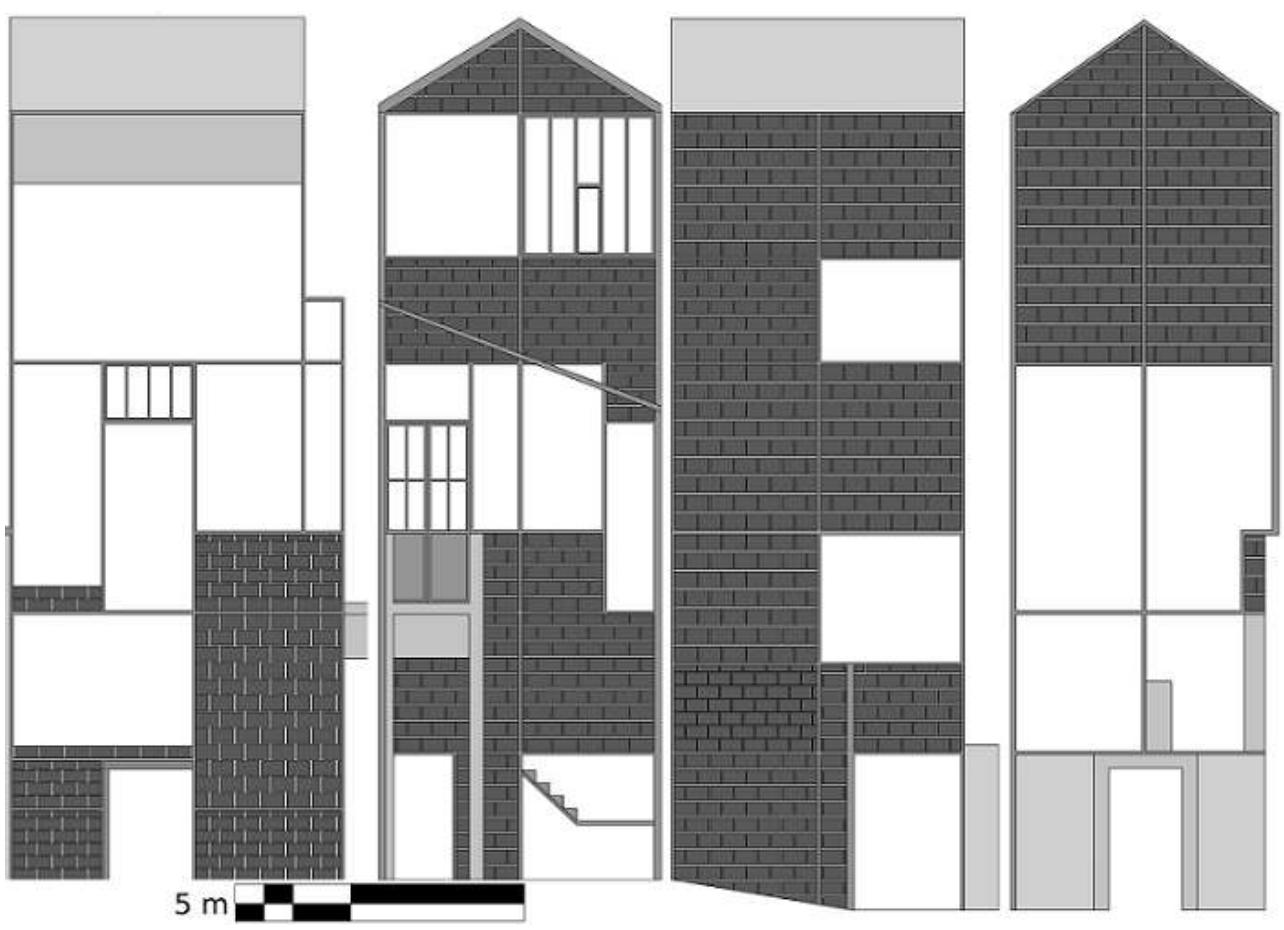

Relevés des façades de gauche à droite est, nord, ouest et sud Relevé Jérôme Féru et Cyril Lacheze ; DAO Cyril Lacheze

Il nous reste à souligner ici la provenance locale des matériaux : si les fermes en acier ont pu être préfabriquées ailleurs et que les machines viennent parfois de l'étranger, la grande majorité des matériaux de construction a été produite au plus près. Les 
moellons utilisés à divers endroits sont du granite et du schiste de même aspect que ceux de la falaise, et il est fort probable qu'ils en aient tout simplement été extraits par dynamitage. Quant aux tuiles (et probablement aux briques), un grand nombre d'entre elles portent le nom de Saint-Fromond, centre important de production de terres cuites architecturales situé à une cinquantaine de kilomètres en aval sur la Vire (Simon 2009). Ainsi, si la conception de l'usine était manifestement pensée en amont de son implantation, les matériaux employés ont été choisis en fonction des contraintes et opportunités sur place, tout comme l'adaptation des structures au terrain.

\section{Organisation interne}

23 Les parties de l'usine dédiées à la production qui sont les seules suffisamment connues pour pouvoir être analysées, étaient, d'après les vestiges matériels, conçues spécifiquement pour une organisation fluidifiée de la production. En effet, toutes les zones sont reliées par des ouvertures ou des rampes d'une largeur minimale de 1,37 m, dimension redondante dans toutes les parties du lieu, qui correspondait probablement à une largeur standardisée de chariots de transport. Les installations industrielles les plus lourdes étaient d'ailleurs situées à proximité de ces voies de circulation.

La fabrication des pièces en elles-mêmes prenait place au rez-de-chaussée d'un bâtiment aujourd'hui rasé au sud de la longère conservée, avant de prendre le montecharge à présent isolé pour subir un contrôle au premier étage. De là, elles empruntaient une passerelle encore en partie visible, exactement de la largeur susdite, pour rejoindre le premier étage de la longère. Dans celui-ci, elles pouvaient subir des traitements de surface ou une peinture, avant de redescendre au rez-de-chaussée par une rampe toujours de la même largeur, puis être stockées et enfin expédiées par camion par deux ouvertures spécialement conçues à cette fin.

Certaines installations sont encore visibles dans l'usine, globalement bien préservées et à leur emplacement d'origine, ou du moins dans la même pièce. L'atelier de galvanoplastie employait des cuves métalliques afin d'effectuer des électrolyses, notamment de cadmium et de chrome-dur ; ces cuves, de plusieurs modules différents, sont encore présentes dans la pièce dédiée quoique renversées et déplacées. Celles-ci étaient semble-t-il disposées sur des bases bétonnées, groupées en trois ensembles longilignes et très étendus, encore en place ; le matériau utilisé était du béton simple ou armé selon l'endroit observé, sans qu'une logique ne soit apparue pour expliquer cette différence (cf. fig.9). Deux machines également liées aux finitions sont d'autre part toujours en place, à savoir un grand four pour faire sécher les pièces traitées, et une machine à peinture. Le four provenait d'une société de pointe située à Aix-en-Provence, signe d'une recherche de matériel de bonne qualité, quitte à le faire venir de loin. La même réflexion peut être effectuée pour ce qui est de l'imposante chaudière de l'usine, elle aussi à son emplacement d'origine. Elle provenait de Belgique. 
Fig.9. Supports de cuves de galvanoplastie

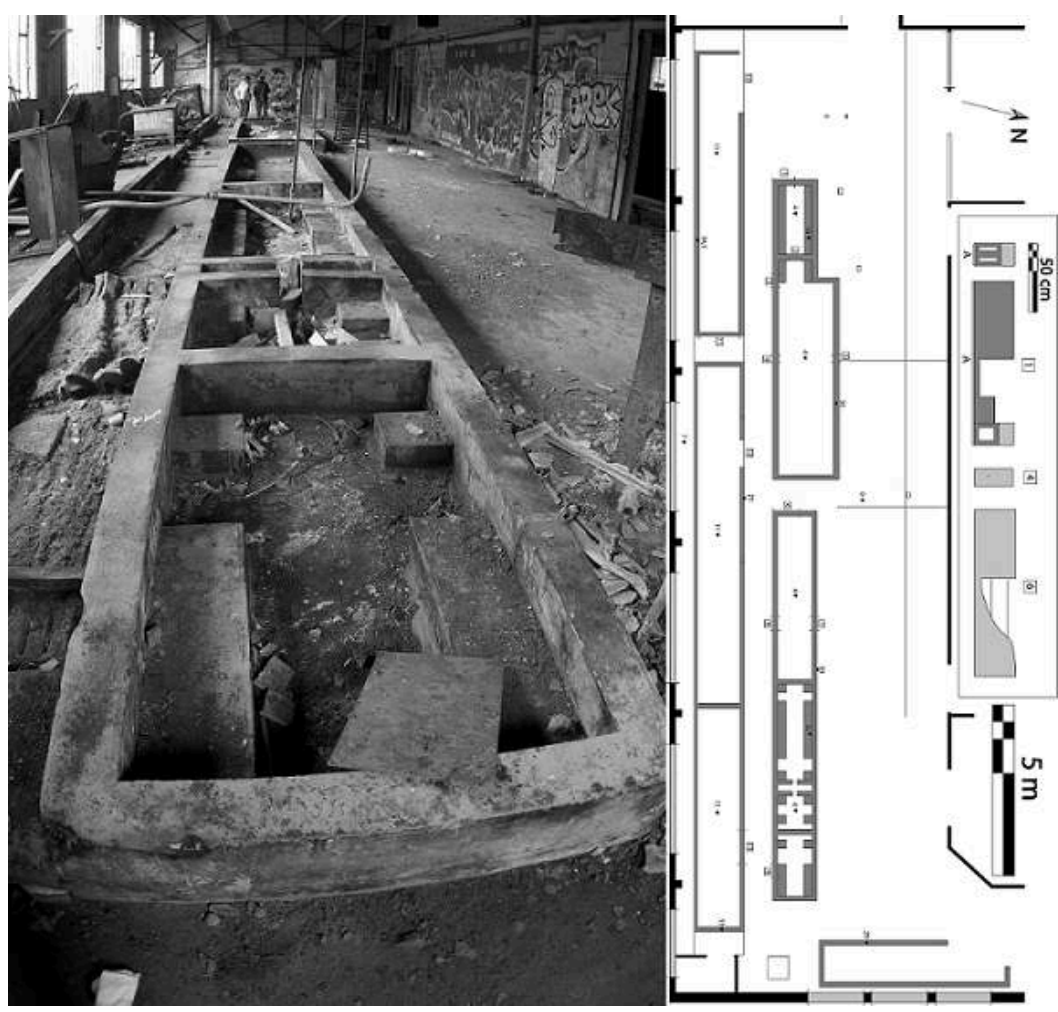

Photographie et relevé en plan. En encadré, des coupes effectuées à différents endroits des bords des cuves, montrant selon le cas une structure en béton simple ou armé.

Photographie Alexis Seydoux. Relevé Jérôme Féru et Cyril Lacheze ; DAO Cyril Lacheze

Il faut enfin souligner une organisation variable dans le temps de certains espaces, qui n'est documentée que par l'archéologie. En effet, au rez-de-chaussée, directement sous l'atelier de galvanoplastie, se trouvent des cuves de traitement des liquides usagés, visant à leur faire perdre leur toxicité par réaction chimique avant évacuation en camion-citerne (explication fournie par l'ancien chimiste de l'usine lors de l'enquête orale). Ce petit espace présente des murs de parpaings qui ne se rencontrent nulle part ailleurs. A contrario, le plan de 1961 et plusieurs ouvriers ayant travaillé anciennement dans l'entreprise font état de la présence de douches à cet endroit, dont nous n'avons trouvé aucune trace. Il apparaît donc que ce secteur a changé d'utilisation à un instant de la vie de l'usine, changement qui n'est pas indiqué dans les sources textuelles et dont l'enquête orale n'a pas retrouvé la trace (les deux états sont évoqués mais non le moment de la transformation), mais qui est formellement attesté par l'enquête archéologique.

\section{La société et les occupants du site}

\section{Approche historique}

Les sources textuelles, issues d'archives ou trouvées sur place, nous renseignent, d'une part sur l'histoire de la société d'équipementier à ses débuts, d'autre part sur l'activité de l'entreprise en tant que telle et sur la vie des ouvriers. La Société Générale d'Équipement installa son siège social et sa première usine à Puteaux en 1926. La grève 
des ouvriers en 1936 incita son directeur Pierre Compte à délocaliser la production en province. Suite à une première expérience à Villemur-sur-Tarn, il concentra son attention sur Vire et concrétisa l'ouverture du site en août 1936. La main-d'œuvre disponible et bon marché, ainsi que la présence du vaste terrain proche du centre urbain, appuyèrent sa décision. Trente salariés commencèrent ainsi à travailler en 1936 dans les locaux de l'ancienne bonneterie. Sous la direction du conducteur de travaux virois René Châtel, et d'après les plans de l'architecte référent de la société, les principaux travaux de construction s'étalèrent entre 1936 et 1940. Au cours des années 1960, la Société Générale d'Équipement devient une division de Précision Mécanique Labinal, Manuvire (Ababsa 2011), le siège social étant alors transféré à Saint-Ouen et M. Bessières devenant Président Directeur Général.

L'activité de l'entreprise se partageait entre les équipements automobiles et aéronautiques. Le secteur de l'automobile commandait essentiellement des filtres à huile Purflux dont le brevet a été déposé par Précision Mécanique Labinal. Nous avons également relevé la fabrication de bancs d'essais utilisés par les garages (contrôle allumage, dynamo, bougies...), des commandes sous volant «Comodo", des chargeurs rapides de batteries (Généréquip) et des disjoncteurs pour les poids lourds Renault dans les années 1950. L'essentiel de la fabrication aéronautique résidait dans les actionneurs tels que des vérins pneumatiques ou électriques et des commandes de trains d'atterrissage. L'autre part de la fabrication intégrait des feux (ailes, queue, feux anticollision), des lampes UV, des faisceaux, des chariots de démarrage et des pièces de tôlerie en aluminium.

Ces pièces équipaient les avions de Nord Aviation et Sud Aviation, puis, suite à leur fusion, ceux de la Société Nationale Industrielle AéroSpatiale (SNIAS) à partir de 1970, renommée l'Aérospatiale en 1978. L'usine a également travaillé pour des équipementiers tels qu'Hispano-Suiza ou Messier, et a équipé des avions à réaction de transport léger militaire pour la société Morane-Saulnier, ainsi que des Fouga destinés à l'entraînement des pilotes de l'armée de l'air (en particulier le Magister). On peut y ajouter des productions liées à l'astronautique, avec des pièces de missiles nucléaires ou de la fusée Ariane. Les machines-outils nécessaires étaient de plus conçues au sein de l'entreprise par le bureau des méthodes, d'après les prototypes de la région parisienne. La chaîne de production se partageait entre le service des Méthodes chargé de la phase d'étude des pièces prototypes, le service de la fabrication assurant la production conforme au cahier des charges, et celui du contrôle technique pour la vérification.

L'importance de l'entreprise, des salaires relativement attractifs à l'échelle locale et une certaine fierté de travailler pour une industrie de pointe, ont mené l'usine à jouer un rôle crucial dans le paysage de l'emploi et de l'industrie viroise, et par extension également dans la sociabilité. En témoigne sa place à part entière dans les festivités locales, telles que le défilé de 1959 à l'occasion de l'inauguration de l'hôtel de ville, dans lequel une maquette de "fusée $»^{8}$ fut placée à l'avant du char. Comme le montre le tableau d'évolution des effectifs (cf. tableau 1), l'entreprise a employé jusqu'à 1200 personnes au plus fort de son activité en 1939. Les chiffres suivent l'accroissement des besoins militaires au début de la Seconde Guerre mondiale, le travail au service de l'occupant, la chute de l'activité suite aux bombardements et la reprise progressive de la production à partir des années 1950. Cette main-d'œuvre reconnue pour ses compétences était convoitée par des entreprises voisines. C'est ainsi que dans les 
années 1960 des techniciens, cadres et ouvriers spécialisés quittèrent la SGE/Labinal pour des salaires plus attractifs proposés chez Cousin Frères à Flers et Jules Germain à Condé-sur-Noireau. Malgré tout, l'entreprise a indéniablement constitué un pôle industriel majeur pour la ville de Vire, insufflant un renouveau dans l'économie locale lors de sa création, tout en donnant lieu à des opportunités d'embauche, de formation et d'ascension professionnelle.

Tableau 1 : Évolution des effectifs entre 1936 et 1985

\begin{tabular}{|l|l|l|l|l|l|l|l|l|}
\hline Année & 1936 & 1939 & 1940 & 1955 & 1957 & 1959 & 1963 & 1985 \\
\hline Effectif & 30 & 1200 & 1100 & 300 & 450 & 543 & 574 & 500 \\
\hline
\end{tabular}

\section{La vie des ouvriers}

Derrière le site désaffecté transparaissent des carrières professionnelles, des équipes de travail et une émulation propre à l'usine. Ces fragments de vies nous sont parvenus par les témoignages des anciens salariés au cours d'une rencontre organisée dans la matinée du 23 avril 2012 par la municipalité, ainsi par une série entretiens privilégiés. De ces échanges ressortent trois axes principaux : l'usine comme poumon économique ; les tremplins professionnels et le pôle de compétence ; le miroir du creuset social et des mutations du monde industriel. Pour le premier, la ville de Vire des années 1930 présentait en effet un paysage industriel dépouillé de son foyer textile prédominant tout au long de la première industrialisation. Les quelques entreprises qui subsistaient ne suffisaient pas à employer l'ensemble des Virois. L'une des premières salariées, ayant travaillé au secteur du contrôle technique, témoignait d'ailleurs que, comme ancienne couturière, il lui était difficile de trouver du travail à cette périodes

L'entreprise d'équipementier représentait par ailleurs une opportunité de travail et de formation. Plusieurs ouvriers attestent de ce tremplin professionnel. Un employé recruté en tant qu'ajusteur en 1957, à l'âge de 17 ans, devint ingénieur par sa formation interne au dessin industriel, à la production d'outillages et à la programmation du matériel de commande numérique ${ }^{10}$. Dans cette industrie de pointe, les ouvriers témoignent de leur mémoire des lieux : les opérations de traitements de surfaces sont déclinées par l'ancien chimiste, l'espace du contrôle technique est décrit par une employée chargée des licences américaines; les évocations de la chaîne de production, des collègues et des supérieurs, de l'ambiance de travail, émaillent les échanges.

La vie de l'usine se fait également le miroir de l'histoire sociale et des mutations inhérentes au domaine industriel. Ce contexte historique et social jaillit à plusieurs reprises lorsque les salariés évoquent la grève de 1958, revendiquant une hausse des salaires de 8\%, le travail aux heures tardives pour les Allemands entre 1942 et 1944, les bombardements de 1944 assénant un coup d'arrêt à la production. Par ailleurs, les employés évoquent le souvenir joyeux des activités et des structures de loisirs développées par des dirigeants de l'usine (clubs sportifs, sorties hebdomadaires à la mer en bus). Le stade municipal de Vire en est un symbole : il fut construit en 1940 par le Président Directeur Général d'alors, Pierre Compte, dont il porte le nom. L'extension de la production à l'automobile a nui au pôle de l'aéronautique. Les restructurations 
des années 1970 ont dispersé les ouvriers dans des antennes annexes, notamment dans la zone industrielle nord de 2,5 hectares. Elles préfiguraient le démantèlement progressif $d u$ site. Les employés émettent d'ailleurs des hypothèses quant aux raisons de la fermeture: diversification de la production prenant le pas sur l'aéronautique, contraintes physiques de la vallée encaissée et limites d'extension, pénurie d'hébergements après la guerre et coûts de transport.

\section{Réoccupation du site}

\section{Étudier la vie des friches industrielles} abandonnée, marquant l'espace principalement par des références musicales; certains occupants allant même jusqu'à y créer deux nouvelles salles réalisées à l'aide de barricades composées de portes et autres éléments architecturaux tombés au sol. Nous pouvons en particulier distinguer des traces faisant référence au mouvement punk et à ses variantes, courant anarchiste communément considéré comme apparu au milieu des années 1970, et dont les protagonistes vont à l'encontre des valeurs établies et prônent la liberté créative. Nous pouvons mentionner le logo du groupe Dead Kennedys, ou encore des têtes dotées de crêtes, certaines déclarant parfois «Oi!», onomatopée faisant référence à un mouvement alternatif du punk et surtout à la musique l'accompagnant; ou bien l'acronyme « $H \& C$ » pour Hardcore, autre sous-genre musical de punk, non loin d'une ligne de texte extraite d'un titre du groupe Fugazi daté de 1991.

Cousine du mouvement punk et apparue dans le même temps dans les classes prolétaires britanniques, la culture skinhead peut également être signalée. 
Originellement d'obédience communiste, antifasciste et antiraciste, elle se devine à travers le logo "RSK" (initiales de Red Skinhead) et un slogan "Destroy Racism" complété d'un dessin où un individu jette une croix gammée dans une poubelle. Un dérivé direct habituellement étroitement associé au terme skinhead, plus techniquement qualifié de bonehead, s'est au contraire tourné vers le néonazisme. Il pourrait apparaitre dans le corpus de graffitis à travers quelques croix gammées (extrêmement brouillonnes toutefois) et dessins à tendance antisémite (étoile de David associée à un phallus). Par ailleurs, autres cousins du punk nettement moins engagés politiquement, une inscription " $\mathrm{AC} / \mathrm{DC}$ » et un pentacle inversé, constituent des références explicites au hard rock ( $\mathrm{AC} / \mathrm{DC}$ étant l'une des plus célèbres formations de cette scène) et heavy metal.

Les punks et leurs variantes ne sont pas les seuls présents parmi les mouvements ayant une influence musicale. Une tête de rasta est représentée sur un mur, non loin d'un tag «Marley »- aucune autre référence à la culture rastafari n'étant présente, il semble probable que les tagueurs n'aient pensé qu'à la musique reggae et non à la portée spirituelle de ce mouvement. Sur quelques murs et au sol se trouvent des références au mouvement hip-hop, lui aussi étroitement associé à un autre genre musical, le rap. Plus éloigné du monde musical cette fois, et de manière plus étonnante en ce qu'il est moins associé à des manifestations graphiques de ce type, le phénomène geek (intimement lié à l'univers informatique et au jeu vidéo) est également particulièrement représenté avec des références telles qu'un personnage de l'univers Mario de Nintendo. L'une des représentations les plus mémorables du lieu semble provenir d'un syncrétisme entre deux mouvements : à l'étage de l'usine, un grand personnage jaune doté d'une crête de punk pourrait être une interprétation d'un Psykokwak, pokemon également proposé par Nintendo et originellement affublé de trois " plumeaux » noirs sur le sommet de la tête (cf. fig.10). La représentation n'est toutefois guère explicite et nous ne pouvons affirmer cette identification. 
Figure 10 : Graffitis sur un mur

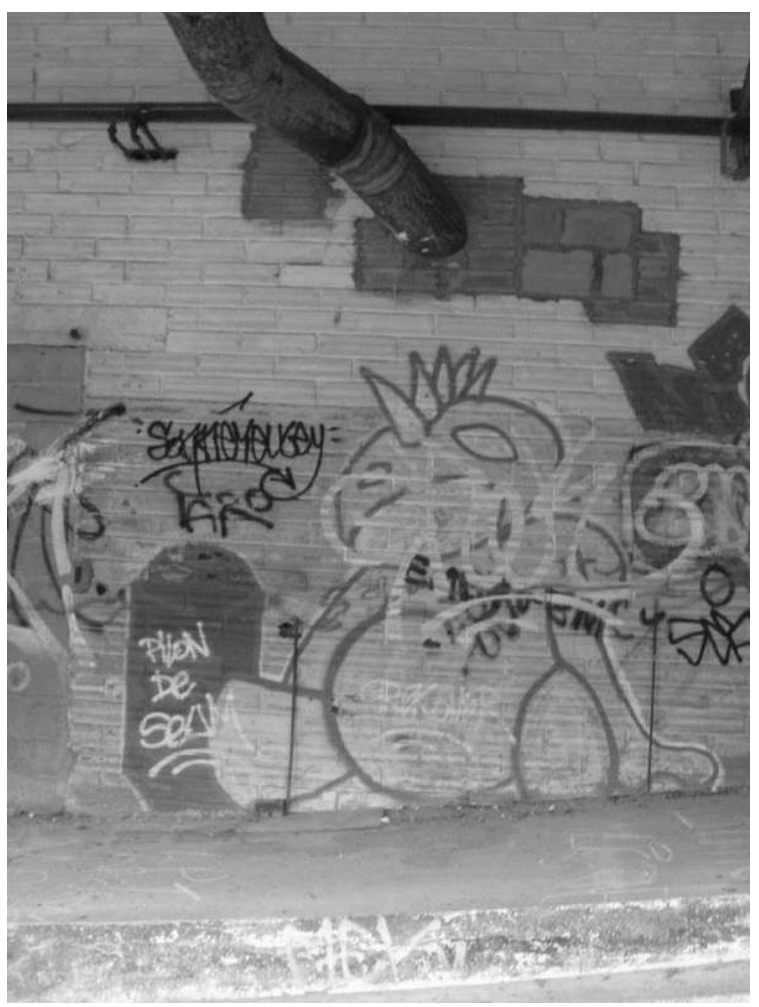

Ces graffitis représentent un certain nombre de mouvements culturels.

Photographie Jérôme Féru

\section{Synthèse et conclusion}

39 L'implantation de la SGE a ainsi eu un impact très fort sur le terrain et la rivière, déjà industrialisés au XIX ${ }^{\mathrm{e}}$ siècle mais dans des proportions bien moindres. Le $\mathrm{XX}^{\mathrm{e}}$ siècle s'est caractérisé par un bétonnage complet du terrain, s'accompagnant par l'accentuation d'une canalisation forcée de la rivière. Cette canalisation modifia quelque peu le cours naturel de la rivière et surtout camoufla les vestiges visibles des occupations antérieures.

L'usine présentait quelques particularités architecturales, construite par rapport au terrain, le rez-de-chaussée voyant un de ses murs réalisé dans la falaise même et des rampes permettant de se déplacer plus facilement entre bâtiments placés à hauteurs différentes. Hormis ces spécificités, la construction est particulièrement classique, avec majoritairement des structures en hangar. Les bâtiments ont gardé la même place dans la chaîne de production au cours des années, malgré quelques modifications constatées par rapport aux plans.

41 Du point de vue sociologique, les anciens salariés éprouvent une fierté manifeste à avoir travaillé au sein de la SGE, par la conscience de posséder un savoir-faire hors du commun, mais également d'avoir appartenu à ce qui pouvait ressembler à une famille élargie (avant les dernières années de l'usine et la crise économique). La jeunesse viroise a également été marquée par cette usine après son abandon (ce qui correspond 
à un tiers de l'existence des lieux), que ce soit pour y faire des manifestations artistiques ou en tant que lieu de réunion clandestin.

Fig.11. Rendu tridimensionnel de la friche

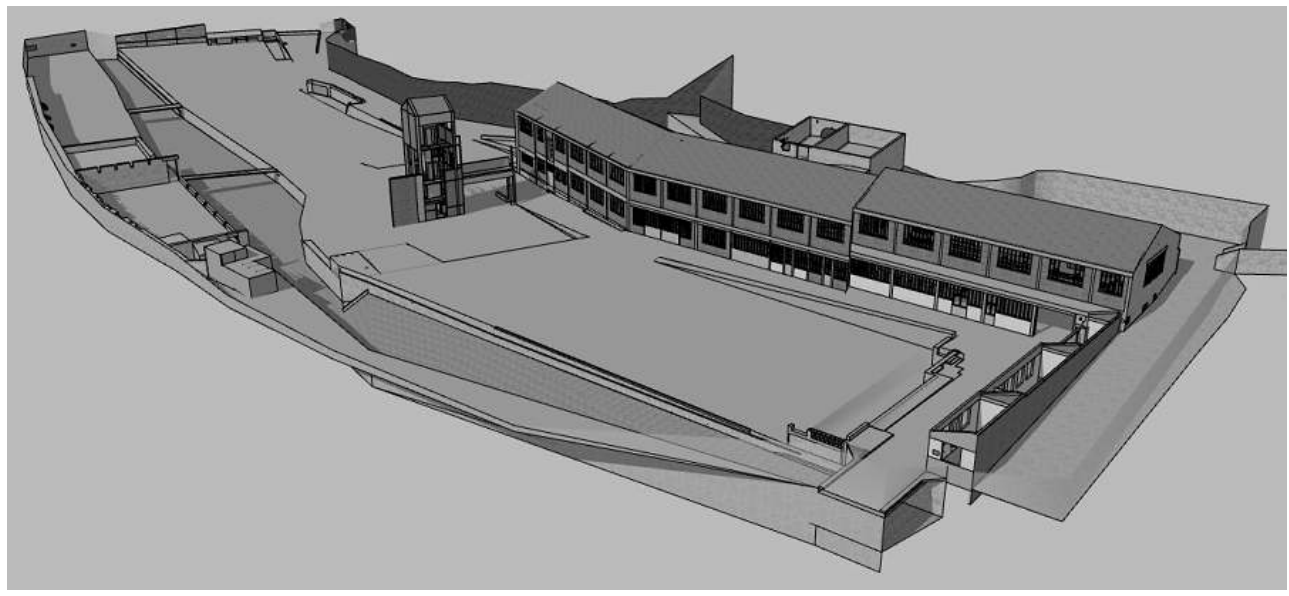

Le rendu a été réalisé sur le logiciel Sketchup, d'après les relevés de terrain.

Relevés Morgane Moëllo, Jérôme Féru et Cyril Lacheze. DAO Cyril Lacheze

Suite à la consultation des anciens salariés par les élus et à la transmission du rapport à l'Atelier des Paysages chargé de proposer des modèles de reconversion et de renaturation du site, le projet d'aménagement retenu suscite des problématiques de cohérence historique et patrimoniale. La solution envisagée, un parc agrémenté de deux moulins fictifs, occulte d'une part les aménagements hydrauliques visibles de l'ancienne filature-bonneterie, et d'autre part la question cruciale de la mémoire collective des lieux, à savoir la problématique initiale de conservation et de valorisation d'éléments de l'usine Société Générale d'Équipement/Labinal. En tout état de cause, les éléments restant de la friche ont été rasés à la pelle mécanique en 2015, et aucune solution de mise en valeur du site n'est actuellement arrêtée. L'étude réalisée ici constitue donc la seule documentation gardant trace de ces bâtiments dans leur état de friche, l'application à leur égard d'une méthodologie archéologique ayant non seulement permis d'en apprendre plus sur leur matérialité, mais également d'en générer un rendu tridimensionnel (cf. fig.11).

\section{BIBLIOGRAPHIE}

ABABSA Malik, « L'histoire de LABINAL à nos jours », La Gazette 3AF Midi-Pyrénées, 2011

BONNOT Thierry, « Une manufacture de céramique vue à travers ses déchets : expérience pluridisciplinaire autour d'un dépotoir », Artefact, 6, 2018, p.11-28 ; DOI : https://doi.org/10.4000/ artefact.781

DAUMAS Maurice, L'archéologie industrielle en France, Paris, Robert Laffont, 1980 
DEONNA Waldemar, L'archéologie. Son domaine, son but, Paris, Flammarion, 1922

HURARD Séverine, ROUMEGOUX Yves, CHAOUI-DERIEUX Dorothée, «L'archéologie à l'épreuve de la modernité. De l'opportunisme à la maturité », Les nouvelles de l'archéologie, 137, 2014, p.3-9

JOURNOT Florence, BELLAN Gilles (dir.), Archéologie de la France moderne et contemporaine, Paris, La Découverte, 2011

MOËLLO Morgane, LACHEZE Cyril, FERU Jérôme, « La Friche des Vaux, Vire (Calvados). Pour une archéologie contemporaine de l'industrie », L'Archéologie industrielle en France, 62, 2013, p.32-34

OFFENSTADT Nicolas, Urbex RDA. L'Allemagne de l'Est racontée par ses lieux abandonnés, Paris, Albin Michel, 2019

RENK Thomas, « A Guide to Recording Structural Details of Historic Building », Historical Archaeology, vol.3, 1969, p.34-48

SIMON Cécile, « Saint-Fromond. Briqueterie du Porribet », 2009 ; URL : https://

journals.openedition.org/adlfi/3741

\section{NOTES}

1. Moëllo Morgane, Lacheze Cyril, Féru Jérôme, Vire (Calvados), Friche industrielle des Vaux, site de la Société Générale d'Équipement/Labinal. Rapport d'étude historique et archéologique, 16 au 27 avril 2012, s.l., s.n., 2013, 2 vol., 404 p.

2. Cette méthodologie de relevé met à profit le fait que les bâtiments industriels emploient généralement des formes géométriques simple, et qu'il est rarement nécessaire de les analyser avec autant de détails que des structures plus anciennes pour appréhender leur agencement. Un croquis des structures est d'abord réalisé - approximativement à l'échelle dans la version de Thomas Renk, sans soin particulier ni réalisme dans la nôtre - figurant les éléments à relever pour obtenir une vue d'ensemble complète des structures, c'est-à-dire avant tout les dimensions des murs. Ces dimensions sont ensuite mesurées à l'aide de simples décamètres et reportées sur le croquis, et seules les éventuelles zones nécessitant un relevé détaillé spécifique font l'objet d'une attention particulière. Les cotations reportées sur le croquis sont ensuite employées pour restituer au propre les relevés à l'échelle des structures (Renk 1969).

3. Toute la chaîne de production y était incluse, de la filature et la tonte des apprêts à l'étendage des draps sur les coteaux des Rames. Archives départementales du Calvados, 3P/1984, Cadastre napoléonien, Vire, 1833. Feuille C2 du Centre, parcelles 593-855, 1/2500 ${ }^{\mathrm{e}}$.

4. Archives départementales du Calvados, S 12896/a, Dossier Juhel-Pondegrenne, 1850-1855.

5. Archives départementales du Calvados, 820W39439 - Document retraçant les évolutions administratives et structurelles de la société, 1963.

6. Photographie aérienne de 1956. Courtoisie des Services Techniques de la ville de Vire.

7. Précision Mécanique Labinal, Usine des Vaux de Vire. Plan masse, 1961. Courtoisie des Services Techniques de la ville de Vire.

8. Le terme fut à l'époque employé pour la décrire dans un petit journal de l'entreprise dont seuls deux numéros nous sont connus. Il était alors d'actualité : si la France venait tout juste de démarrer un programme spatial, la « course à l'espace » battait son plein entre les États-Unis et l'URSS (les satellites Spoutnik et Explorer 1 avaient été lancés en 1957 et 1958, et l'actualité était aux survols de la Lune avec les programmes Pioneer et Luna). La maquette en question était toutefois dotée d'ailes et évoque plutôt, pour des yeux actuels, un avion supersonique. Vire Volt, $\mathrm{n}^{\circ} 1$, février 1959. Courtoisie de Maurice Chédemail.

9. Émilienne Soimier, contrôle technique, 1936-1977, entretien du 27 avril 2012, Vire. 
10. Pierre Desjardins, contrôle technique - dessins industriel, 1957-1963, entretien du 26 avril 2012, Vire.

11. "Urbex » pour " exploration urbaine », ainsi définie par Nicolas Offenstadt : "visite sans autorisation et le plus souvent sans but lucratif de lieux délaissés ou abandonnés » (Offenstadt 2019).

12. Il ne s'agit pas d'une généralité absolue, d'où notre précision de nature géographique : les friches japonaises par exemple, bien qu'extrêmement nombreuses et également visitées par des « urbexeurs » ou des marginaux, demeurent pour beaucoup en excellent état.

13. Ou sous cultures, dans le sens anglo-saxon de subculture. Quel que soit le terme employé, nous désignons par celui-ci des cultures ciblées, avec des références et des codes propres, mais sans notion de hiérarchie ou d'" infériorité " par rapport à la culture " générale » formée de l'ensemble des éléments culturels communs à l'ensemble d'une société donnée.

\section{RÉSUMÉS}

Située à Vire (Calvados, France), dans le quartier de Vaux de Vire, l'usine SGE, active de 1936 à 1985, a constitué un acteur majeur de la vie industrielle de cette localité normande de taille moyenne, avant de devenir pendant une trentaine d'années une friche industrielle. Le site a été démoli récemment. Nous avons eu l'occasion de le documenter quelques années avant sa disparition, avec une triple approche : analyse archéologique des bâtiments ; étude historique de documents écrits, la plupart retrouvés au sein de la friche; enquête orale auprès des anciens employés de la société. Cette approche transdisciplinaire a souligné l'intérêt d'une démarche archéologique dans un contexte pourtant très récent. L'archéologie, en effet, d'une part apporte des éléments de compréhension qui complètent efficacement les apports des deux autres méthodes et d'autre part, permet de documenter la vie du site après son abandon

Located in Vire (Calvados, France), in the Vaux de Vire district, the SGE factory, which was active from 1936 to 1985, was a major player in the industrial life of this medium-sized Norman town, before becoming an industrial wasteland for some thirty years. The site was recently demolished. We had the opportunity to document it a few years before its disappearance, with a threefold approach: archaeological analysis of the buildings; historical study of written documents, most of which were found within the wasteland; and an oral survey of the company's former employees. This transdisciplinary approach underlined the interest of an archaeological approach in a very recent context. Archaeology, in fact, on the one hand provides elements of understanding that effectively complement the contributions of the other two methods, and on the other hand, makes it possible to document the life of the site after its abandonment.

\section{INDEX}

Mots-clés : histoire des techniques, archéologie, archéologie industrielle, patrimoine industriel, friche, pluridisciplinarité, urbex

Keywords : history of technology, archaeology, industrial archaeology, industrial heritage, interdisciplinarity, urban archaeology

Thèmes : Entretiens et leçons 


\section{AUTEURS}

\section{MORGANE MOËLLO}

Historienne des techniques de formation, Morgane Moëllo s'est spécialisée dans l'étude et la valorisation du patrimoine technique et industriel. En tant que conservatrice du patrimoine, elle a repris la direction de l'Écomusée Creusot Montceau au cours de l'année 2019. Son champ d'expertise porte sur l'impact technique et environnemental des aménagements industriels, en particulier hydrauliques, du XVIII ${ }^{\mathrm{e}}$ siècle à nos jours.

\section{CYRIL LACHEZE}

Cyril Lacheze est docteur en histoire des techniques et ATER en histoire des sciences à l'Université Paris 1 Panthéon-Sorbonne. Formé parallèlement en archéologie, il s'est spécialisé dans les deux disciplines sur les périodes moderne et contemporaine, s'intéressant en conséquence à l'archéologie contemporaine actuellement en développement en France. Dans le cadre de sa thèse, il a appliqué les méthodes développées sur le site de Vire, objet de cet article, sur des fours à terre cuite architecturale du XIX ${ }^{\mathrm{e}}$ siècle.

\section{JÉRÔME FÉRU}

Archéologue de formation, Jérôme Féru est technicien de fouilles sur des chantiers archéologiques depuis 2009. Après avoir travaillé dans le domaine de la médiation culturelle, il est aujourd'hui agent opérationnel pour l'Institut National de Recherches Archéologiques Préventives (INRAP). 